\title{
A New Convertible Authenticated Encryption Scheme Based on Bilinear Square Diffie-Hellman Problem
}

\author{
Han-Yu Lin ${ }^{+}$ \\ Department of Computer Science and Engineering, National Taiwan Ocean University, Taiwan
}

\begin{abstract}
Convertible authenticated encryption (CAE) scheme is a cryptographic scheme which has been found numerous practical applications like on-line credit card transactions, confidential contract signing and the protection of digital evidence, etc. In this paper, we propose a new CAE scheme based on the bilinear square Diffie-Hellman problem. The proposed scheme is proved secure against adaptive chosen-plaintext attacks (CPA2) and adaptive chosen-message attacks (CMA) in the random oracle mode. Compared with previous schemes, ours not only provides better functionalities, but also has provable security.
\end{abstract}

Keywords: convertible, authenticated encryption, digital signature, bilinear pairings, random oracle

\section{Introduction}

In 1994, Horster et al. [1] introduced an authenticated encryption (AE) scheme simultaneously combining the functions of digital signature and public key encryption. That is, the requirements of authenticity and confidentiality [2] are both satisfied. In such a scheme, a signer can produce an authenticated ciphertext while only a designated recipient having the corresponding private key can decrypt it and verify the embedded signature. Yet, when a designated recipient encounters the situation of later repudiation, he cannot convince anyone of signer's dishonesty. To deal with the dispute, in 1999, Araki et al. [3] addressed a variant providing an additional arbitration mechanism. However, Zhang and Kim [4] pointed out that Araki et al.'s scheme cannot withstand a universal forgery attack.

In 2002, Wu and Hsu [5] came up with a convertible authenticated encryption (CAE) scheme allowing the designated recipient to solely announce a converted signature. The next year, Huang and Chang [6] proposed another enhanced variant. Nevertheless, Lv et al. [7] showed that neither the Wu-Hsu nor the Huang-Chang schemes achieve the security requirement of confidentiality. In 2009, Lee et al. [8] further introduced the ElGamal-based CAE scheme. In 2012, Lu et al. [9] introduced a convertible multiauthenticated encryption scheme for generalized group communications. In 2014, an RSA-based CAE scheme [10] is also addressed. In this paper, we propose a new CAE scheme from bilinear pairing cryptosystems. The proposed scheme is proved secure in the random oracle model.

\section{The Proposed Scheme}

In this section, we present our proposed scheme from bilinear pairings. The used notations are stated as Table 1. The proposed CAE scheme consists of the following algorithms:

$\operatorname{Setup}\left(1^{k}\right)$ : On input a security parameter $k$, the Setup algorithm selects two groups $\left(\boldsymbol{G}_{1},+\right)$ and $\left(\boldsymbol{G}_{2}, \times\right)$ of the same prime order $q$. Let $P$ be a generator of order $q$ over $\boldsymbol{G}_{1}, e: \boldsymbol{G}_{1} \times \boldsymbol{G}_{1} \rightarrow \boldsymbol{G}_{2}$ a bilinear pairing and $h_{1}$ : $\{0,1\}^{k} \times \boldsymbol{G}_{1} \rightarrow Z_{q}, h_{2}: \boldsymbol{G}_{1} \times \boldsymbol{G}_{1} \times \boldsymbol{G}_{2} \rightarrow\{0,1\}^{k}$ and $h_{3}: \boldsymbol{G}_{1} \rightarrow \boldsymbol{G}_{1}$ collision resistant hash functions. The algorithm outputs public parameters params $=\left\{\boldsymbol{G}_{1}, \boldsymbol{G}_{2}, q, P, e, h_{1}, h_{2}, h_{3}\right\}$.

\footnotetext{
+ Corresponding author. Tel.: +886-2-2462-2192 ext 6656; fax: +886-2-2462-3249.

E-mail address: hanyu@mail.ntou.edu.tw.
} 
Reg_U(i): On input an index $i$, the Reg_U algorithm chooses a private key $x_{i} \in Z_{q}$, computes the public key $Y_{i}=x_{i} P$ and then further generates the public key certificate Cert $_{i}$ by the X.509 standard [11].

TABLE I: THE USED NOTATIONS

\begin{tabular}{|l|l|}
\hline$\left(\boldsymbol{G}_{1},+\right)$ & Additive group of prime order $q$ \\
\hline$\left(\boldsymbol{G}_{2}, \times\right)$ & Multiplicative group of prime order $q$ \\
\hline$Z_{q}^{*}$ & multiplicative group of integers modulo $q$ \\
\hline$x \in Z_{q}^{*}$ & element $x$ in set $Z_{q}^{*}$ \\
\hline$x \leftarrow Z_{q}^{*}$ & sampling element $x$ uniformly in set $Z_{q}^{*}$ \\
\hline$|x|$ & bit-length of integer $x$, also absolute value of $x$ \\
\hline$\oplus$ & logical operation XOR \\
\hline $\operatorname{Pr}[E]$ & probability of event $E$ occurring \\
\hline
\end{tabular}

Sign_M $\left(m, x_{s}, Y_{v}\right)$ : On input a message $m$, the public key $Y_{v}$ of the designated recipient and the private key $x_{s}$ of signer, the algorithm chooses $t \in Z_{q}^{*}$ to compute $R=t P, \sigma=\left(x_{s}+h_{1}(m, R)\right)^{-1} R, W=h_{3}\left(t Y_{v}\right), Z=$ $e\left(x_{s} Y_{v}, W\right), r=m \oplus h_{2}(R, \sigma, Z)$ and then outputs the authenticated ciphertext $\delta=(R, \sigma, r)$.

$\operatorname{Verify\_ AEC}\left(\delta, x_{v}, Y_{s}\right)$ : On input an authenticated ciphertext $\delta=(R, \sigma, r)$, the private key $x_{v}$ of designated recipient and the public key $Y_{s}$ of signer, the algorithm first computes $W=h_{3}\left(x_{v} R\right)$ and $Z=e\left(x_{v} Y_{s}\right.$, $W)$ to recover the message $m$ as $m=r \oplus h_{2}(R, \sigma, Z)$ and then checks the redundancy embedded in $m$. The algorithm further verifies the signature by checking whether $e\left(\sigma, Y_{s}+h_{1}(m, R) P\right)=e(R, P)$. If it holds, the message $m$ and its converted signature $\Omega=(R, \sigma)$ is outputted; else, the error symbol $\perp$ is returned as a result. We prove that the equality works correctly. From the left-hand side of it, we have $e\left(\sigma, Y_{S}+h_{1}(m, R) P\right)$ $=e\left(\left(x_{s}+h_{1}(m, R)\right)^{-1} R, Y_{s}+h_{1}(m, R) P\right)=e\left(\left(x_{s}+h_{1}(m, R)\right)^{-1} R,\left(h_{1}(m, R)+x_{s}\right) P\right)=e(R, P)$ which leads to the right-hand side of it.

\section{Security Proof and Comparison}

In this section, we first state the underlying security assumption and prove the security of our scheme.

Bilinear Square Diffie-Hellman Problem; BSDHP: Given an instance $(P, A, B) \in \boldsymbol{G}_{1}$ where $P$ is a generator, $A=a P$ and $B=b P$ for some $a, b \in Z_{q}^{*}$, compute $e(P, P)^{a^{2} b} \in \boldsymbol{G}_{2}$.

Bilinear Square Diffie-Hellman (BSDH) Assumption: For every probabilistic polynomial-time algorithm $A$, every positive polynomial $Q(\cdot)$ and all sufficiently large $k, A$ can solve the BSDHP with the advantage at most $1 / Q(k)$, i.e., $\operatorname{Pr}\left[A(P, a P, b P)=e(P, P)^{a^{2} b} ; a, b \leftarrow Z_{q}{ }^{*}, P, a P, b P \leftarrow \boldsymbol{G}_{1}\right] \leq 1 / Q(k)$. The probability is taken over the uniformly and independently chosen instance and over the random choices of $A$.

Theorem 1. (Proof of Confidentiality) The proposed CAE scheme is secure against adaptive chosenplaintext attacks (CPA2) in the random oracle model if there exists no probabilistic polynomial-time adversary that can break the BSDHP with non-negligible advantage.

Proof: Suppose that a probabilistic polynomial-time (PPT) adversary $A$ can break our scheme with nonnegligible advantage $\varepsilon$ under the adaptive chosen-plaintext attack after marking at most $q_{h_{i}} h_{i}$ (for $i=1$ to 3 ), $q_{\text {Reg_U }}$ Reg_U and $q_{\text {Sign_M }} \operatorname{Sign} \_M$ queries. Then we can construct another algorithm $B$ to obtain $e(P, P)^{a^{2} b}$ by taking the $(P, a P, b P)$-BSDHP instance as inputs. In this proof, $B$ simulates a challenger to $A$.

Setup: $B$ runs the $\operatorname{Setup}\left(1^{k}\right)$ algorithm and sends public parameters params $=\left\{\boldsymbol{G}_{1}, \boldsymbol{G}_{2}, q, P, e\right\}$ to $A$.

Phase 1: $A$ issues the following kinds of queries adaptively:

- $h_{1}(m, R)$ oracle: $B$ chooses $v_{1} \in_{R} Z_{q}$, adds the entry $\left(m, R, v_{1}\right)$ into $h_{1}$-list and returns $v_{1}$ as a result.

- $h_{2}(R, \sigma, Z)$ oracle: $B$ first searches the $h_{2}$-list for an matched entry; else, $B$ seeks the form ( $R, \sigma$, NULL, $\left.v_{2}\right)$ and then replaces NULL with $Z$. Otherwise, $B$ chooses $v_{2} \in_{R}\{0,1\}^{k}$, adds the entry $(R, \sigma$, $\left.Z, v_{2}\right)$ into $h_{2}$-list and returns $v_{2}$ as a result.

- $h_{3}\left(t Y_{v}\right)$ oracle: $B$ chooses $v_{3} \in_{R} \boldsymbol{G}_{1}$ and adds the entry $\left(t Y_{v}, v_{3}\right)$ into $h_{3}$-list. Finally, $B$ returns $v_{3}$ as a result. 
- $\quad$ Reg_U query $\langle i\rangle$ : If $i=I D s, B$ returns $\left(Y_{s}=a P, C_{e r t}\right)$. If $i=I D v, B$ returs $\left(Y_{v}=b P\right.$, Cert $\left._{v}\right)$. Otherwise, $B$ runs Reg_U $\langle i\rangle$ and then returns $\left(Y_{i}\right.$, Cert $\left._{i}\right)$ to $A$.

- Sign_M query $\left\langle m, Y_{i}, Y_{j}\right\rangle$ : If $Y_{i} \neq a P, B$ returns $\operatorname{Sign} \_\mathrm{M}\left(m, x_{i}, Y_{j}\right)$. When $Y_{i}=a P, B$ chooses $t, v_{1} \in_{R}$ $Z_{q}$ and $v_{2} \in_{R}\{0,1\}^{k}$, computes $\sigma=d P, r=m \oplus v_{2}$ and $R=d(a P)+v_{1} d P$, adds the entry $\left(m, R, v_{1}\right)$ into $h_{1}$-list and the entry $\left(R, \sigma\right.$, NULL, $\left.v_{2}\right)$ into $h_{2}$-list. Then the ciphertext $\delta=(R, \sigma, r)$ is returned to A.

Challenge: $A$ generates two messages, $m_{0}$ and $m_{1}$, of the same length. $B$ flips a coin $\lambda \leftarrow\{0,1\}$ and chooses $t, v_{1} \in_{R} Z_{q}, \sigma^{*} \in_{R} \boldsymbol{G}_{1}$ and $v_{2} \in_{R}\{0,1\}^{k}$, computes $r^{*}=m_{\lambda} \oplus v_{2}$ and $R^{*}=t P$ and adds the entry $(t(b P)$, $a P)$ into $h_{3}$-list and the entry $\left(R^{*}, \sigma^{*}\right.$, NULL, $\left.v_{2}\right)$ into $h_{2}$-list. The ciphertext $\delta^{*}=\left(R^{*}, \sigma^{*}, r^{*}\right)$ is then delivered to $A$ as a target challenge. A can make new queries as those stated in Phase 1.

Output: Finally, $B$ randomly chooses an entry of $h_{2}$-list and outputs $Z$ as a correct answer to the BSDHP.

Analysis of the game: To win the game with a non-negligible advantage, $A$ might attempt to decrypt the ciphertext $\delta^{*}=\left(R^{*}, \sigma^{*}, r^{*}\right)$ and recover $m_{\lambda}$. Since $B$ sets $h_{3}\left(t Y_{v}\right)=h_{3}\left(x_{v} R^{*}\right)=a P$ and implicitly defines $h_{2}\left(R^{*}\right.$, $\left.\sigma^{*}, Z^{*}\right)=v_{2}$ where $Z^{*}=e\left(x_{v} Y_{s}, W\right)=e(P, P)^{a^{2} b}, B$ has a non-negligible advantage to solve the BSDHP on condition that $A$ makes an $h_{2}\left(R^{*}, \sigma^{*}, Z^{*}\right)$ oracle query in phase 2 . The probability that $A$ guesses the correct random value without asking an $h_{2}$ oracle is not greater than $2^{-k}$, i.e., the probability that $Z^{*}$ is in the $h_{2}$-list is not less than $\left(\varepsilon-2^{-k}\right)$. Since $B$ randomly chooses an entry from the $h_{2}$-list and outputs $Z$ as the answer, we have $\operatorname{Pr}\left[Z=Z^{*}\right]=q_{h_{2}}{ }^{-1}$. Consequently, $B$ can solve the BSDHP with a non-negligible advantage $\left(q_{h_{2}}{ }^{-1}\right)(\varepsilon-$ $\left.2^{-k}\right)$ in polynomial-time.

Theorem 2. (Proof of Unforgeability) The proposed CAE scheme is secure against existential forgery on adaptive chosen-message attacks (CMA) in the random oracle model if there exists no probabilistic polynomial-time adversary that can break the BSDHP with non-negligible advantage.

Proof: Suppose that a PPT adversary A can break the proposed scheme with non-negligible advantage $\varepsilon$ under the adaptive chosen-message attack after making at most $q_{h_{i}} h_{i}$ (for $i=1$ to 3), $q_{R_{\text {Reg }} U}$ Reg_U and $q_{S i g n_{\_} M}$ Sign_M queries. Then we can construct another algorithm $B$ to obtain $e(P, P)^{a^{2} b}$ by taking the $(P, a P, b P)$ BSDHP instance as inputs. In this proof, $B$ simulates a challenger to $A$.

Setup: $B$ runs the $\operatorname{Setup}\left(1^{k}\right)$ algorithm and sends public parameters params $=\left\{\boldsymbol{G}_{1}, \boldsymbol{G}_{2}, q, P, e\right\}$ to $A$.

Phase 1: $A$ adaptively makes new queries as those defined in Theorem 1 . Note that in the $j$-th $h_{3}\left(t Y_{v}\right)$ oracle query where $j \leq q_{h_{3}}, B$ directly returns $W_{j}=a P$.

Forgery: $A$ outputs a forged authenticated ciphertext $\delta^{*}=\left(R^{*}, \sigma^{*}, r^{*}\right)$ for some $m^{*}$.

Output: Finally, $B$ randomly chooses an entry of $h_{2}$-list and outputs $Z$ as a correct answer to the BSDHP.

Analysis of the game: If $A$ computes $Z^{*}$ with the $j$-th result of $h_{3}\left(t Y_{v}\right)$ oracle query, i.e., $Z^{*}=e\left(x_{s} Y_{v}, W_{j}\right)$ $=e\left(x_{s} Y_{v}, a P\right)=e(P, P)^{a^{2} b}$ and the forged authenticated ciphertext $\delta^{*}=\left(R^{*}, \sigma^{*}, r^{*}\right)$ is valid, the value $Z^{*}$ should be kept in some entry of the $h_{2}$-list when $A$ makes an $h_{2}\left(R^{*}, \sigma^{*}, Z^{*}\right)$ oracle query. The probability that $A$ guesses the correct random value without asking an $h_{2}$ oracle is not greater than $2^{-k}$, i.e., the probability that $Z^{*}$ is in the $h_{2}$-list is not less than $\left(\varepsilon-2^{-k}\right)$. Since $B$ has set the $j$-th $h_{3}\left(t Y_{v}\right)$ oracle query to be $a P$ and randomly outputs $Z$ from some entry of the $h_{2}$-list as the answer, we obtain $\operatorname{Pr}\left[Z=Z^{*}\right]=1 / q_{h_{2}}$ and $\operatorname{Pr}\left[W=W_{j}\right]$ $=1 / q_{h_{3}}$. Therefore, the advantage to solve the BSDHP is $\left(\varepsilon-2^{-k}\right)\left(q_{h_{2}} q_{h_{3}}\right)^{-1}$.

We compare the proposed scheme with some previous ones including AUI [3], Sek [12] and WH [5] schemes. Detailed comparisons in terms of functionalities and security are demonstrated as Table 2.

\section{Conclusions}

CAE schemes have played an important role in e-commerce and the protection of digital evidence, etc. In the literature, we proposed a new CAE scheme based on the bilinear square Diffie-Hellman problem. Unlike previous works which only provide heuristic security proofs, we formally prove that the proposed scheme is secure in the random oracle mode. When a later dispute occurs, the designated recipient can solely reveal the converted signature to convince any third party of the signer's dishonesty. Also, we still preserve the merit that the signature conversion process takes no extra efforts. With better functionalities and the provable security, we claim that the proposed scheme has crucial benefits to practical applications. 
TABLE II: COMPARISONS OF THE PROPOSED AND PREVIOUS SCHEMES

\begin{tabular}{|c|c|c|c|c|}
\hline Item \& Scheme & AUI & Sek & WH & Ours \\
\hline Non-interactive conversion process & $\sqrt{ }$ & $\sqrt{ }$ & $\sqrt{ }$ & $\sqrt{ }$ \\
\hline Unforgeability/Non-repudiation/No conversion cost & $\times$ & $\times$ & $\sqrt{ }$ & $\sqrt{ }$ \\
\hline Confidentiality \& Forward secrecy & $\sqrt{ }$ & $\sqrt{ }$ & $\times$ & $\sqrt{ }$ \\
\hline Provable security & $\times$ & $\times$ & $\times$ & $\sqrt{ }$ \\
\hline
\end{tabular}

\section{Acknowledgements}

This work was supported in part by the Ministry of Science and Technology of Republic of China under the contract number MOST 104-2221-E-019-018.

\section{References}

[1] P. Horster, M. Michel and H. Peterson, "Authenticated encryption schemes with low communication costs," Electronics letters, 1994, 30 (15): 1212-1213.

[2] W. Stallings, Cryptography and Network Security: Principles and Practices, 4th Ed., Pearson, 2005.

[3] S. Araki, S. Uehara and K. Imamura, "The limited verifier signature and its application," IEICE Transactions on Fundamentals, 1999, E82-A (1): 63-68.

[4] F. Zhang and K. Kim, "A universal forgery on Araki et al.'s convertible limited verifier signature scheme," IEICE Transactions on Fundamentals, 2003, E86-A (2): 515-516.

[5] T. S. Wu and C. L. Hsu, "Convertible authenticated encryption scheme," The Journal of Systems and Software, 2002, 62 (3): 205-209.

[6] H. F. Huang and C. C. Chang, "An efficient convertible authenticated encryption scheme and its variant," Proceedings of the 5th International Conference on Information and Communications Security (ICICS2003), Springer-Verlag, Berlin, 2003, pp. 382-392.

[7] J. Lv, X. Wang and K. Kim, "Practical convertible authenticated encryption schemes using self-certified public keys," Applied Mathematics and Computation, 2005, 169 (2): 1285-1297.

[8] C. C. Lee, M. S. Hwang and S. F. Tzeng, "A new convertible authenticated encryption scheme based on the ElGamal cryptosystem," International Journal of Foundations of Computer Science, 2009, 20 (2): 351-359.

[9] C. F. Lu, C. L. Hsu and H. Y. Lin, "Provably convertible multi-authenticated encryption scheme for generalized group communications," Information Sciences, 2012, 199 (15): 154-166.

[10] T. S. Wu and H. Y. Lin, "Provably secure proxy convertible authenticated encryption scheme based on RSA," Information Sciences, 2014, 278 (10): 577-587.

[11] ISO/IEC 9594-8, Information technology - open systems interconnection - the directory: public-key and attribute certificate frameworks, International Organization for Standardization, 2001.

[12] M. R. Sekhar, "Signatures scheme with message recovery and its applications," International Journal of Computer Mathematics, 2004, 81 (3): 285-289. 